\title{
International Severe Weather and Flash Flood Hazard Early Warning Systems-Leveraging Coordination, Cooperation, and Partnerships through a Hydrometeorological Project in Southern Africa
}

\author{
Robert Jubach ${ }^{1, *}$ and A. Sezin Tokar ${ }^{2}$ \\ 1 Hydrologic Research Center, 12555 High Bluff Drive, San Diego, CA 92130, USA \\ 2 U.S. Agency for International Development, Office of U.S. Foreign Disaster Assistance, \\ Ronald Reagan Building, Washington, DC 20523, USA; stokar@usaid.gov \\ * Correspondence: rjubach@hrcwater.org; Tel.: +1-858-461-4560; Fax: +1-858-704-4955 \\ Academic Editors: Sharon B. Megdal and Susanna Eden \\ Received: 20 February 2016; Accepted: 12 June 2016; Published: 20 June 2016
}

\begin{abstract}
Climate, weather and water hazards do not recognize national boundaries. Transboundary/regional programs and cooperation are essential to reduce the loss of lives and damage to livelihoods when facing these hazards. The development and implementation of systems to provide early warnings for severe weather events such as cyclones and flash floods requires data and information sharing in real time, and coordination among the government agencies at all levels. Within a country, this includes local, municipal, provincial-to-national levels as well as regional and international entities involved in hydrometeorological services and Disaster Risk Reduction (DRR). Of key importance are the National Meteorological and Hydrologic Services (NMHSs). The NMHS is generally the authority solely responsible for issuing warnings for these hazards. However, in many regions of the world, the linkages and interfaces between the NMHS and other agencies are weak or non-existent. Therefore, there is a critical need to assess, strengthen, and formalize collaborations when addressing the concept of reducing risk and impacts from severe weather and floods. The U.S. Agency for International Development/Office of U.S. Foreign Disaster Assistance; the United Nations World Meteorological Organization (WMO); the WMO Southern Africa Regional Specialized Meteorological Center, hosted by the South African Weather Service; the U.S. National Oceanic and Atmospheric Administration/National Weather Service and the Hydrologic Research Center (a non-profit corporation) are currently implementing a project working with Southern Africa NMHSs on addressing this gap. The project aims to strengthen coordination and collaboration mechanisms from national to local levels. The project partners are working with the NMHSs to apply and implement appropriate tools and infrastructure to enhance currently operational severe weather and flash flood early warning systems in each country in support of delivery and communication of warnings for the DRR entities at the regional, national and local levels in order to reduce the loss of life and property.
\end{abstract}

Keywords: early warning; hydrometeorological hazards; severe weather; flash flood guidance; disaster risk reduction

\section{Introduction}

The following article overviews the framework of a project currently underway in Southern Africa designed to reduce the risks from severe weather and flash floods in the region. The stated goal of the project is to improve accuracy, lead time, communication and dissemination of severe weather and flash flood warnings to communities. The project aims to (1) strengthen the capacity of National 
Meteorological and Hydrologic Services (NMHSs) to provide timely and effective early warnings of extreme hydrometeorological events (with appropriate lead times for taking action); and (2) develop products and information that address needs of users such as National Disaster Management Agencies, Non-Governmental Organizations and the general public to reduce the loss of lives and livelihoods and to protect property and the environment in the Southern Africa region.

At this point the project is not mature enough to measure its effectiveness and so we do not provide any detailed statistics or information in this regard. There are plans to evaluate the project as well as the impact of improved forecasts and early warnings-these plans are discussed in Section 5. The project is presented here at this time because the authors believe that this project is a unique approach to improving early warnings on a regional basis through the application of existing operational systems that provide data and information for extreme weather and flash floods to all countries in the region. We believe that a regional approach to using actual, proven operating systems as a catalyst for the NMHS and supporting governmental agencies to fully develop their early warning potential and address the critical end-to-end early warning system components and requirements will prove most successful.

\section{Background}

Southern Africa is among the most vulnerable regions of the world to hydrometeorological hazards including floods, cyclones, droughts and extreme temperatures. According to the Center for Research on the Epidemiology of Disasters [1], 72\% of natural disasters reported in the countries in the Southern Africa region were severe weather and hydrometeorological in nature (e.g., floods, flash floods, storms, droughts, extreme temperatures). As a result of a United Nations World Meteorological Organization (WMO) survey [2] and from discussions at various regional hydrometeorological hazard workshops conducted by the Hydrologic Research Center (HRC), it was noted that all countries in the region report being impacted in some way by the effects of these hazards. In a recent workshop organized by the WMO, the HRC and the U.S. Agency for International Development/Office of U.S. Foreign Disaster Assistance (USAID/OFDA), meteorological and hydrologic forecasters and disaster managers from Botswana, Lesotho, Malawi, Mozambique, Namibia, South Africa, Swaziland, Zambia, and Zimbabwe stated that flash floods/floods, heavy precipitation, strong winds, and thunderstorms were the most prevalent hydrometeorological hazards affecting their countries [3].

A recent example includes the major flash flooding and riverine flooding that occurred in Malawi, Mozambique, Madagascar, and Zimbabwe from December 2014 to January 2015. This flooding affected more than 135,000 people in the four countries. Malawi was especially hit hard, with flooding in over half of the country's districts [4].

The Sendai Framework for Disaster Risk Reduction: 2015-2030 (SFDRR) that was adopted by 187 nations aimed at the substantial reduction of disaster risk and losses in lives, livelihoods and health and in the economic, physical, social, cultural and environmental assets of persons, businesses, communities and countries. As outlined in the principles and priorities under SFDRR, a critical component of Disaster Risk Reduction (DRR) is to enable early action. One way achieve this is through a well-functioning early warning system that delivers accurate, reliable and understandable warnings, in a timely manner, to authorized disaster managers and populations at risk. Effective early warning systems involve four operational components requiring close cooperation and coordination among the all agencies involved. The United Nations International Strategy on Disaster Reduction (UNISDR) [5] defined the four components including:

1. Monitoring and Warning Service-Develop hazard monitoring and early warning services (observing, detecting, monitoring and forecasting hazards, and developing warning messages);

2. Risk Knowledge-Systematically collect data on hazards and vulnerability and undertake risk assessments (assessing the potential risks and integrating risk information in the warning messages);

3. Dissemination and Communication-Communicate risk information and early warnings (distributing, rapidly and reliably, understandable warnings to authorities, disaster managers and 
the population at risk, with levels of warning that are linked to levels of preparedness, readiness and emergency operations); and,

4. Response Capability-Build national and community response capabilities (community-based emergency planning, preparedness and training programs to ensure effective response to warnings to reduce the potential impacts).

Of primary importance for early warning systems is that they are truly end-to-end in nature. An end-to-end system consists of a warning and response system where the components are interconnected. Each component in this process is critical to reduce the impacts of hydrometeorological extremes and provide essential lead times to aid decisions. Failure of one component will lead to failure of the entire system to save lives and livelihoods. State-of-the-art technology and a perfect forecast will not save lives if the populations at risk are not informed in a timely manner or do not have plans and policies in place in advance to reduce impacts. Well-prepared communities remain vulnerable to these hazards if they do not have access to and understand information that provides the lead time needed to take necessary actions. Communications and warning/information dissemination are important attributes of a successful warning system as well as investment into building the capacity of forecasters and user knowledge. In addition, close coordination must occur between all sectors and between national and local governments for systems to function properly with clear lines of roles and responsibilities to avoid confusion and chaos during disasters. Important parts of this process are the systems that enable user feedback to periodically improve to address the needs of decision makers.

As our focus in this article is on severe weather and hydrologic/hydrometeorological hazards, the primary agencies we are concerned with that must deal with early warnings for these hazards are the NMHSs. The role of the NMHSs is critical; the majority of natural disasters are weather related as discussed during the previously referenced workshop [3]. Flash floods, floods, heavy precipitation, drought, strong winds, thunderstorms (including lightning and hail), tropical storms, extreme temperatures, heat/cold waves, wildfires, fog, hazardous seas/lakes, snow, and storm surges are common hazards that are a threat to lives and livelihoods in Southern African countries.

Rogers and Tsirkunov [6] outlined the importance of sustainable partnerships and commitment to cooperation and information exchange between NMHSs with disaster management agencies for early warning systems. These partnerships must be supported by legal authority and institutional arrangements for the successful development and sustainability of effective early warning systems. As they have noted, partnerships are important in two ways: early warning tasks in an end-to-end system are not usually managed by a single entity, and they provide the ability to leverage resources and provide opportunities to share costs, knowledge, and lessons learned. Most significantly, effective partnerships can ensure a consistent message from multiple sources (e.g., severe weather and flash flood warnings and information). To be effective partners, the NMHSs must understand the decision-making process made by all sectors affected by the hazard to ensure that information is tailored to the specific needs of the user. This includes:

- Providing and communicating efficient, clear and timely weather- and warning-related data and information;

- Understanding the hazard's effect on the user operations and needs that vary from user to user and event to event at different temporal and spatial scales;

- Having a quantitative understanding of the social and economic cost and benefit of the warnings;

- Understanding the decisions that depend on the warning and the lead time; and,

- Understanding and conveying the uncertainty in the warning.

Over the years, international and regional entities have worked on strengthening the different components of early warning systems and DRR. This has included the development of specialized technical capacities for monitoring, detecting, modeling and forecasting for different natural hazards, legal and institutional aspects, emergency preparedness and response planning for communities, 
coordination of humanitarian response, and public education. In some countries, hazard-specific early warning systems have been developed. Yet the success of some of these programs has been mixed. Often what are needed to integrate all the various components of early warning systems are projects which focus on actual operational systems that act as a catalyst for the NMHS and supporting governmental agencies to fully develop their early warning potential and address the critical early warning system components and requirements as noted above. One such project, now fully underway in Southern Africa, deals with both severe weather-related hazards (a meteorological issue) and flash floods (a hydrometeorological issue). This project, known as the Delivery of Warnings of Hydrometeorological Hazards-Southern Africa Region is discussed in the following sections.

\section{Southern Africa Region Project Overview}

USAID/OFDA along with the WMO, RSMC Pretoria (WMO Regional Specialized Meteorological Center, managed by the South African Weather Service), the U.S. National Oceanic and Atmospheric Administration/National Weather Service (NOAA/NWS) and the HRC have partnered to implement a project to link and enhance the operational Southern Africa Severe Weather Forecast Demonstration Project (SWFDP) and the Southern Africa Region Flash Flood Guidance (SARFFG) systems with the objective of leveraging those systems to provide improved service delivery of forecasts, warnings and information to the DRR agencies/organizations at the regional, national and local levels in Southern Africa. The project includes the Southern African countries of Botswana, Lesotho, Malawi, Mozambique, Namibia, South Africa, Swaziland, Zambia, and Zimbabwe. It was kicked off with workshops in Pretoria, South Africa, conducted by the project partners and attended by representatives from all nine countries [3].

Both the SWFDP and the SARFFG are regional-based systems that have been designed to provide forecasters at the NMHSs the necessary data and information to provide timely and effective warnings of severe hydrometeorological events, including conditions for rainfall that could lead to flash floods. Because they are regional-based systems hosted by a Regional Center (RSMC Pretoria), data, information and forecast products can be generated efficiently and cost-effectively, thereby complementing the limited capacity available at national levels. Both systems encompass end-to-end early warning system concepts by including the elements of (1) observing, detecting and assisting forecasters to develop hazard forecasts and warnings; (2) assessing the potential risks and integrating risk information in the warning messages; and (3) distributing, rapidly and reliably, understandable warnings to authorities, risk managers and the population at risk as well as many other users, such as the agricultural sector. Linking the two systems both programmatically and technically enhances their capabilities to address a fourth element of an end-to-end early warning system-emergency preparedness and response to warnings at all relevant levels (national to local) to minimize the potential impacts of extreme hydrometeorological events. (Additional discussion regarding both systems is provided below.)

This project: (1) integrates data from both the operational SARFFG and SWFDP systems; (2) improves forecast operations at RSMC Pretoria and the nine Southern African countries that have access to both systems; (3) builds capacity of the hydrological and meteorological operations of each NMHS and RSMC Pretoria; (4) improves service delivery to the National and Regional Disaster Management agencies and DRR users through improved warning dissemination (e.g., lead time, message content and impact information); and, (5) enhances severe weather disaster awareness, preparedness and response. For the DRR sector in each country, the degree of outreach and readiness is assessed. This includes the legal frameworks in effect to assure effective use of improved warnings and forecasts.

An essential aspect of this project is having the DRR community clearly define the information and products they need to maximize response and minimize loss of life and property. This is best done through prototyping and feedback, as the DRR community must understand the forecast uncertainties well in order to provide useful information back to the NMHSs. This is because of the high uncertainties 
of the forecasts in small regions that requires risk management conditional on the local forecast uncertainties (rather than based only on a priori knowledge).

For this project to deliver improved services, it integrates/links and enhances the two fully operational systems, builds capacity through coordinated training and develops and implements a severe weather/flash flood Concept of Operations (CONOPS) and Roadmap for each country for warning services to connect to disaster response operations and users.

Linkages between the systems will lead to improvements in the capabilities of both initiatives and create closer collaborations between NMHSs, leading to improved service delivery. In essence these two systems have been implemented in parallel with similar concepts of operation, basic approach, and region (southern Africa). At the same time, they have distinct differences in relation to both the temporal and spatial nature of each extreme hydrometeorological event and on the emphasis of the hazards, respectively, which require different approaches. Each separate system could benefit from programmatic and technical linkages, including, for example:

- Shared outputs from each program's planning/steering committee meetings;

- Joint workshops that focus on using products from either system for DRR;

- A common format for alert/warning protocols;

- Joint validation programs;

- Joint access to system products (e.g., rainfall estimates and forecasts);

- Hypertext linked access to each system; and,

- Collaborations between system forecasters for the development of consistent forecast and outlook discussions relevant to both systems.

A particular emphasis will be placed on "reaching the last mile", making sure warnings developed from both systems reach the affected population and users in a timely and accurate manner and are in a format that can be easily understood and acted upon to achieve maximum value in resulting disaster response actions. Improved linkage between NMHS warning products and services to national and community disaster response activities is the principal goal for integrating these two successful forecast and warning systems.

\subsection{Severe Weather Forecasting Demonstration Project (SWFDP)}

In essence the SWFDP is a process whereby scientists from global and regional centers work with severe weather forecasters at the national level to identify services that would assist the national disaster response and risk reduction efforts, and which can be implemented almost immediately by tailoring numerical weather prediction model outputs and other forecasting tools that exist in the most advanced centers, making them routinely available at the national level. The majority of NMHSs are not able to develop or run the weather forecast models due to limited capacity and resources. The SWFDP employs a 'Cascading Forecasting Process' whereby outputs from forecast systems that are available and free in advanced global centers are cascaded to NMHSs through a designated regional center which provides interpretation and guidance on severe weather from the next few hours (nowcasting) to the next five days (short- to medium-range forecasting). This allows forecasters at the NMHSs to focus limited resources on considering the impact of this weather in their country, service delivery, and communicating the message to users in their countries to ensure timely and effective early warnings and protective responses. The SWFDP project includes building the capacity of national meteorologists in the application of the cascaded information and in the development of services to meet the disaster management communities' needs. Opportunities are taken to involve disaster managers and the news media in preparation of the user requirements.

The SWFDP has improved the lead time and reliability for alerts about high-impact events such as heavy precipitation, strong winds, and damaging waves. It has strengthened NMHS interaction with disaster management and civil protection agencies, local communities and media. The SWFDP is making a major contribution to SFDRR and is contributing to the Sustainable Development Goals [7] as well as climate change adaptation. More broadly, the project is benefiting society in terms of safety of both the population and operations, including DRR, and in the performance of key socio-economic 
areas, including agriculture, fisheries, aviation, water resource management, health and marine transportation, where meteorological prediction is crucial.

\subsection{Southern Africa Region Flash Flood Guidance System (SARFFG)}

The SARFFG was designed and developed for use by meteorological and hydrologic forecasters in the Southern Africa region (specifically the countries of Botswana, Lesotho, Malawi, Mozambique, Namibia, South Africa, Swaziland, Zambia and Zimbabwe). The primary purpose of the SARFFG is to provide operational forecasters and disaster management agencies with real-time informational guidance products pertaining to the threat of small-scale flash flooding throughout the region. The SARFFG provides the necessary products to support the development of warnings for flash floods from rainfall events through the use of satellite-based rainfall estimates and hydrologic models.

The SARFFG outputs are made available to users (forecasters) to support their analysis of weather-related events that can initiate flash floods (e.g., heavy rainfall, rainfall on saturated soils) and then to make a rapid evaluation of the potential for a flash flood at a location. To assess the threat of a local flash flood, the SARFFG is designed to allow product adjustments based on the forecaster's experience with local conditions, the incorporation of other information (e.g., Numerical Weather Prediction output) and any last-minute local observations (e.g., non-traditional rain gauge data) or local observer reports. The system can be used in its real-time mode or in a forecast mode when outputs are used along with nowcasting and Numerical Weather Prediction precipitation forecasts. The system supports evaluations of the threat of flash flooding over hourly to six-hourly time scales.

Important technical elements of the SARFFG system are the development and use of a bias-corrected satellite precipitation estimate field and the use of land-surface hydrologic modeling. The system then provides information on rainfall and hydrologic response, the two important factors in determining the potential for a flash flood. The system is based on the concept of flash flood guidance and flash flood threat. Both indices provide the user with the information needed to evaluate the potential for a flash flood, including assessing the uncertainty associated with the data.

The flash flood guidance approach to developing flash flood warnings rests on the real-time comparison of observed or forecasted rainfall volume of a given duration and over a given catchment to a characteristic volume of rainfall for that duration and catchment that generates bank full flow conditions at the catchment outlet. If the observed or forecasted rainfall volume is greater than the characteristic rainfall volume then flooding in the catchment is likely (a flash flood threat). The characteristic rainfall volume for a particular catchment and duration, called flash flood guidance, depends on the catchment and drainage network characteristics and the soil water deficit determined by antecedent rainfall, evapotranspiration and groundwater loss [8-10].

The SARFFG system was an important tool in developing and issuing warnings for the previously mentioned flash flooding in Malawi in January 2015. The Malawi Department of Climate Change and Meteorological Services (DCCMS) applied the system to issue timely and accurate warnings to communities impacted by the flooding. The DCCMS used the flash flood guidance and rainfall forecasts from the system to develop the warnings [11]. Lessons learned from events such as the Malawi flooding event plus evaluations of the addition of data and information from the SWFDP system during such events will be factored in to the Southern Africa Region Project in order to further improve the development and dissemination of warnings.

\section{Roadmap and CONOPS Development}

On the operational front, effective early warning systems require the establishment of Roadmap and CONOPS documents by the NMHS to ensure coordination and operational cooperation among the responsible agencies and, for this project, a focus on the SWFDP and SARFFG linked systems. The Roadmap will outline objectives, deliverables, work plans, and organizational makeup. The CONOPS will provide a clear outline of the flow of activities necessary to produce routine and non-routine operations, data/information flow, responsibilities, and ongoing training programs for the linked systems; included are linkages to all aspects of the end-to-end processes that comprise EWS. The development of these two documents by the countries will be accomplished under the guidance 
of the project team-USAID/OFDA, WMO, RSMC, NOAA/NWS, and the HRC. The Roadmap and CONOPS development is a critical element of this project as these documents will be used as input to country-specific implementation plans. The implementation plan then provides a mechanism to track progress in the full integration of the SWFDP and SARFFG systems into NMHS operations and the development of the end-to-end early warning systems for hydrometeorological hazards, specifically for severe weather and flash floods.

\subsection{Roadmap Development}

One of the important aspects of this project is the development of a Roadmap (framework) outlining the actions required to help meet the objective to provide improved service delivery of forecasts, warnings and information to the DRR agencies/organizations at the regional, national and local levels in Southern Africa. This Roadmap is a tool to recognize existing and needed relationships between these assets and the skills, technologies and requirements to meet future needs and goals. This Roadmap integrates product development and training outcomes from this project and accounts for the end-to-end early warning system process.

The various steps and information used in the development of the country roadmaps are as outlined below.

1. Current NMHS Capabilities-Provide a clear and unambiguous understanding of the current conditions of the capabilities to support an end-to-end early warning system for severe weather and flash floods such that appropriate timely and accurate warnings and information can be provided to the affected population.

2. Required Outcomes-In evaluating the required outcomes, the following are to be considered [6]:

- The governance and institutional arrangements necessary to support the development and sustainability of effective early warning systems;

- Appropriate and available technical and operational capacities for observing, detecting, monitoring, forecasting and warning of hazards;

- What constitutes effective partnerships between stakeholders;

- Efficient, clear and timely communication of weather- and warning-related data and information;

- A clear understanding of the decision-making process made by all sectors affected by the hazard to ensure that information is tailored to the specific needs of the user, including:

$\bigcirc \quad$ Understanding the effect of weather and warning-related data and information on the user operations and needs

$\bigcirc \quad$ Knowing the operations and needs for each stakeholder for a given event, which could vary widely

$\bigcirc$ Quantitative understanding of the social and economic cost and benefit of the warnings

- An understanding of and ability to convey the uncertainty in the warning

$\bigcirc$ Understanding the decisions that depend on the warning

Level of acceptance of false alarms (positives or negatives)

- Good communication is important for an effective warning system.

3. Gap Analysis-An important step is to undertake a gap analysis to identify areas needing improvement between the current capabilities and the required outcomes. Critical systems to include in the analysis are observation networks, data/information tools, technical operating procedures/protocols (e.g., SWFDP/SARFFG operations manuals), meteorological and hydrologic forecasting infrastructure, communication infrastructure to ensure data and information reach the target users, and internal and user capacity building. 
Roadmap Activities-Table 1 provides examples of roadmap activities for the NMHSs.

Table 1. Roadmap activities (NMHS responsibilities).

\footnotetext{
Review, Develop Disaster Management Policies

* Analyze existing national- to community-level disaster management plans, procedures and protocols for emergency situations.

* Analyze existing linkages/collaborations between providers and users of severe weather and flash flood warnings.

* Evaluate mandates/roles of the National Meteorological and Hydrologic Service (NMHS) and other national to community disaster management agencies/organizations (what can be sent and to whom by the NMHS).

* Develop partnerships between all stakeholders.
}

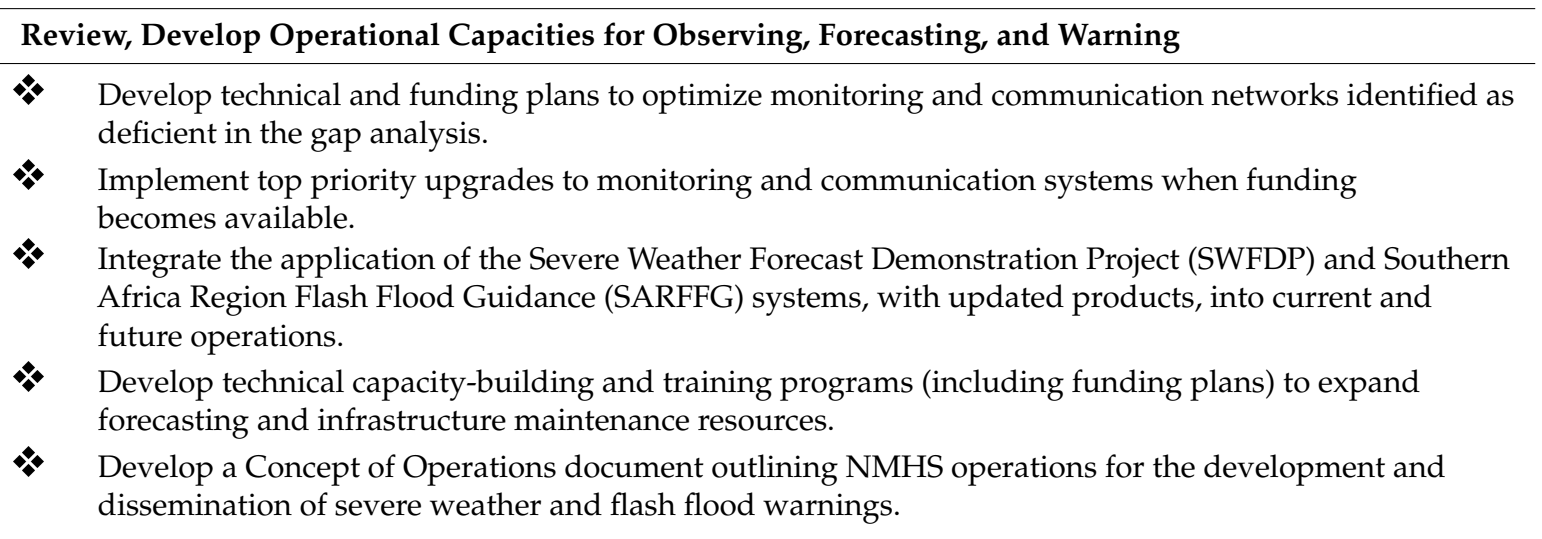

\section{Develop Guidance Products and Protocols}

Assess the extent to which existing severe weather and flash flood warning services are able to meet user demands and requirements.

* Create a working platform to bring together providers and users of severe weather and flash flood warnings to promote better understanding of the diverse and evolving needs of the user groups, especially the last mile, to jointly identify, design, and implement strategies and actions to improve responsiveness to warning services. This platform (which could include stakeholder workshops) will address content, format, delivery, lead time, communication of technical limitations (including uncertainty), literacy and gender.

* Develop appropriate products and data and information packages for users; utilize existing and new products from the SWFDP and SARFFG systems as applicable.

* Create capacity-building and training programs for users of severe weather and flash flood warnings to improve user product development, delivery, usability, evaluation and interpretation.

* Perform continuous assessments of issues (potential or actual) that could affect the NMHS warning services; test feasible options to mitigate the issues.

\footnotetext{
Develop Communication and Dissemination Mechanisms

* Develop technical and funding plans to optimize communication infrastructure identified as deficient in the gap analysis.

* Develop and implement a disaster managers' web-based portal.

* Develop protocols for the dissemination of severe weather and flash flood warnings and other specialized forecasts and NMHS products exchanges with target users.

* Develop protocols for improving warning dissemination through formal and informal channels, including feedback mechanisms before, during, and after an event.

* Implement simulation exercises annually with users to test the communication and dissemination systems for severe weather and flash flood warnings, including feedback mechanisms.
} 


\subsection{CONOPS Development}

A CONOPS document is critical and provides a clear outline of the flow of activities necessary to produce routine and non-routine operations, data/information flow, responsibilities, and ongoing training. A CONOPS describes the likely operation of a future or existing system in the terminology of its users [12]. The countries are in the process of developing an integrated CONOPS document for the operations of both the SWFDP and SARFFG systems for their application to an end-to-end early warning system, including accounting for the linkages between the two systems. Included are references to all aspects of the end-to-end processes that comprise an early warning system and ensure integration into current and future NMHS forecast operations. The CONOPS documents provide the top-level technical specifications and functionality of the early warning system, as well as its look and feel.

Key elements in the Southern Africa country CONOPS documents include:

1. Description of the early warning system;

2. Operations of the early warning system

a integrating products from the SWFDP and SARFFG systems

b integrating local data and information

c information flow;

3. Responsibilities of operational components
a forecast centers
b national, provincial, local national disaster management centers;

4. Responsibilities of support infrastructure-IT and other technical support components;

5. NMHS routine forecast and warning operations

a SARFFG and SWFDP product overview

b meteorological, hydrologic, and precipitation forecast evaluations

c SARFFG and SWFDP product evaluations

d routine bulletins-e.g., guidance, discussions, dissemination protocols

e alerts/warnings_e.g., disaster management, public, dissemination protocols

f validation;

6. Staff training;

7. NMHS non-routine operations-e.g., operations during unusual events; and

8. Outreach
a public
b disaster managers.

\section{Project Evaluation}

The project described in this article is underway and it will take several years to determine its effectiveness. During this time the project team will be working with the countries to monitor and measure its effectiveness, especially with regards to improved service delivery to NMHS users such as DRR organizations. Future activities will involve the review and monitoring of outputs from the application of the Roadmap and Concept of Operation activities to determine if improvements have been made with early warnings for extreme hydrometeorological events. This evaluation will also include a determination of the need for future improvements and/or modifications in operational procedures. In addition, approaches to forecast and warning verification will be documented so they 
can be applied consistently by participating NMHSs. Warning verifications are especially critical and, with the cooperation of the countries, statistics can be derived for accuracy, timeliness, and warning lead times. For forecast verifications, the countries will be trained on the application of a WMO document which will be revised to accommodate SARFFG products under this project [13]. The evaluation will also include assessing the effectiveness and accuracy of the SWFDP and SARFFG systems as tools to support NMHS operations.

Current plans are to have these verifications presented, reviewed and evaluated at a series of steering committee meetings and/or SWFDP/SARFFG technical workshops for discussion, leading to recommendations for further improvements to the end-to-end early warning system including emergency preparedness and response measures.

Of particular interest to the project team, other than the forecast and warning verification statistics, will be feedback to assess the following success metrics:

- $\quad$ Receive positive user responses as to the timeliness and effectiveness of severe weather and flash flood warning products developed by the NMHS using the SWFDP and SARFFG systems and other available (e.g., local) data.

- Success stories that demonstrate reduction in economic losses and lives saved through either the mitigation or aversion of severe weather or flash flood disasters as a direct consequence of improvements in warnings delivered through the NMHS.

\section{Conclusions}

In the developing or least developed countries in Southern Africa, many National Meteorological and Hydrologic Services have limited or no capacity to provide early warning services for extreme hydrometeorological events, including severe weather and flash flood alerts and warnings. There are some NMHSs which have a limited warning capacity but are facing challenges in implementing the currently existing systems available to them. The currently implemented project, Delivery of Warnings of Hydrometeorological Hazards-Southern Africa Region, looks to link and leverage the operational Southern Africa Flash Flood Guidance System and the Severe Weather Forecast Demonstration Project to provide the catalyst for the NMHS and supporting governmental agencies needed to integrate all the various components of early warning systems to fully develop their early warning potential. A key element of the project is the development of Roadmap and CONOPS documents by each country as input to country-specific project implementation plans. These documents are used to track progress in the full integration of the SWFDP and SARFFG systems into NMHS operations and the development of the end-to-end early warning systems for hydrometeorological hazards, specifically for severe weather and flash floods.

Acknowledgments: The authors would like to thank Paul Pilon, Ayhan Sayin, and Abdoulaye Harou of the World Meteorological Organization, Curt Barrett of the U.S. Agency for International Development/Office of U.S. Foreign Disaster Assistance, and Eugene Poolman of the South African Weather Service for their involvement in the development and implementation of this important project for Southern Africa. As part of the Project Management Team, each was involved in the development of the project concept and implementation and work plans critical for a successful project. A special thanks to Konstantine Georgakakos and Eylon Shamir of the Hydrologic Research Center for their review and comments on the article.

Conflicts of Interest: The authors declare no conflict of interest.

Disclaimer: The program was made possible through support provided by the Office of U.S. Foreign Disaster Assistance, U.S. Agency for International Development, under the terms of Award No. AID-OFDA-A-11-00016. The opinions expressed in this publication are those of the authors and do not necessarily reflect the views of the U.S. Agency for International Development.

\section{References}

1. Guha-Sapir, D.; Below, R.; Hoyois, P. EM-DAT: International Disaster Database; Université Catholique de Louvain: Brussels, Belgium, 2016. 
2. United Nations World Meteorological Organization. Capacity Assessment of National Meteorological and Hydrological Services in Support of Disaster Risk Reduction-Analysis of the 2006 WMO Disaster Risk Reduction Country-Level Survey; World Meteorological Organization: Geneva, Switzerland, 2008.

3. United Nations World Meteorological Organization. Delivery of Warnings of Hydrometeorological Hazards-Southern Africa Region-Regional Technical Meeting/CONOPS Workshop, Pretoria, South Africa, 26-30 October 2015, Final Report; World Meteorological Organization: Geneva, Switzerland, 2015.

4. ReliefWeb. Southern Africa Floods-January 2015; U.N. Office for the Coordination of Humanitarian Affairs: Geneva, Switzerland, 2015.

5. United Nations International Strategy for Disaster Reduction. Global Survey of Early Warning Systems; Third International Conference on Early Warning: Bonn, Germany, 2006.

6. Rogers, D.; Tsirkunov, V. Implementing Hazard Early Warning Systems; World Bank Global Facility for Disaster Reduction and Recovery: Washington, DC, USA, 2011.

7. United Nations. Transforming Our World: The 2030 Agenda for Sustainable Development. Available online: http://www.un.org/pga/wp-content/uploads/sites/3/2015/08/120815_outcome-document-ofSummit-for-adoption-of-the-post-2015-development-agenda.pdf (accessed on 17 June 2016).

8. Carpenter, T.; Sperfslage, J.; Georgakakos, K.; Sweeney, T.; Fread, D. National Threshold Runoff Estimation Utilizing GIS in Support of Operational Flash Flood Warning Systems. J. Hydrol. 1999, 224, 21-44. [CrossRef]

9. Georgakakos, K. Analytical Results for Operational Flash Flood Guidance. J. Hydrol. 2006, 317, 81-103. [CrossRef]

10. Georgakakos, K.; Graham, R.; Jubach, R.; Modrick, T.; Shamir, E.; Spencer, C.; Sperfslage, J. Global Flash Flood Guidance System Phase I; HRC Technical Report No. 9; Hydrologic Research Center: San Diego, CA, USA, 2013.

11. Graham, R., Ed.; Flash Flood Guidance Gazette; Hydrologic Research Center: San Diego, CA, USA, 2015; Volume 5.

12. University Corporation for Atmospheric Research. Flash Flood Early Warning System Reference Guide; ISBN: 978-0-615-37421-5. University Corporation for Atmospheric Research: Boulder, CO, USA, 2010.

13. World Meteorological Organization. Forecast Verification for the African Severe Weather Forecasting Demonstration Projects; WMO Doc-No. 1132; World Meteorological Organization: Geneva, Switzerland, 2014.

(C) 2016 by the authors; licensee MDPI, Basel, Switzerland. This article is an open access article distributed under the terms and conditions of the Creative Commons Attribution (CC-BY) license (http:/ / creativecommons.org/licenses/by/4.0/). 